\title{
Research on Three-source Layout of Active Decoy against ARM
}

\author{
Fan Zhenqin, Wang Yongjie, Li Bo, Liu Heng \\ No. 63981 Unit of PLA, Wuhan, China \\ email:phoe_nirvana@163.com
}

\begin{abstract}
Based on the active decoy principle and analysis on fire-control radar working characteristic, a fire-control radar active decoy layout model covering the anomalistic layout is designed in this paper. By simulation, the relationship between decoying success rate and the radar's position in decoy system is obtained, and the recommended configuration of three-source decoy system is also put forward.
\end{abstract}

Keywords: active decoy; layout; decoy effect

\section{Introduction}

In current, active decoy is the most effective and common mean to antagonize anti-radiation missile. The active decoys can radiate the same signal as radar and form a new illusive energy center to induce anti-radiation missile (ARM). By this, radar is protected, as in [1] and [2]. Fire-control radar is the key of current air defense. It can obtain the moving trace of air target by continuous track radiation and send it to fire-control unit to attack. Compared to warning radar, fire-control radar is easier to be attacked because of its unique work mode. It is very necessary to equipment active decoys for fire-control radar. And the layout of fire-control active decoy should be studied to support its application in battlefield.

By a large of researches and making design reality into consideration, domestic scholars made a certain conclusion that three-source layout is the most appropriate mean for warning radar. In spite of the difference work mood, fire-control radar and warning radar have the same actual problems in choosing the number of active decoys. Three-source active decoy system is chosen as the study object and simulated in this paper.

Three-source layout can be divided into two parts: inerratic layout and anomalistic layout. When the distance between radiation sources has some certain relationship or their line connecting angle is fixed, like isosceles triangle layout and right triangle layout, the layout is called inerratic layout. When there is no constraint, the layout is called anomalistic layout. Currently, domestic scholars have made many researches. Two three-source inerratic layouts, right triangle layout and equilateral triangle layout, are studied in [3], and the conclusion that three-source equilateral triangle layout is the optimal layout. The 
appropriate distance between resources under three-source equilateral triangle layout is obtained by simulation in [4]. But these conclusions are not sufficient. Because the anomalistic layout is not taken into consideration and there is no proof that inerratic layout is better than anomalistic layout. And in real battles, Limited by the geography environment, anomalistic layout have more application space than inerratic layout. Making anomalistic layout fully considered, a new decoy layout model is designed in this paper. By changing radar's position in whole decoy system, all layout shapes are included in this model. The relationship between decoying effect and radar's position is obtained by simulation and analysis.

\section{Fire-control radar active decoy principle}

Passive microwave homing technology is adopted in ARM to accomplish the precision guidance for radiation. The key point of ARM is the seeker. It is a broadband passive single pulse tracking system. It has very good anti-interference ability for single radiation source. But when there are more than one radiation sources in its resolution angle, it will not track any one of the radiation sources, but their energy center. This is the basic principle of active decoy system against ARM.

Assume the decoy system is composed of one radar and $n$ decoys. Take radar as the origin and establish the coordinate. The space location of radiation sources and ARM in this coordinate is shown in Fig. 1.

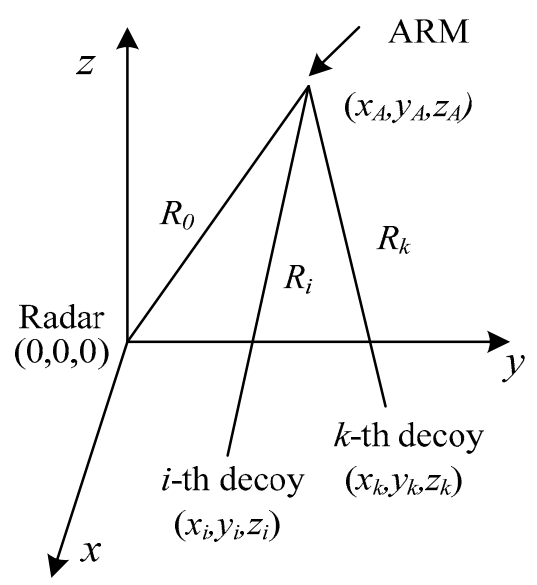

Figure 1. Schematic diagram of decoy system.

As the seeker of ARM is tracking the normal direction of electromagnetic field wave front, the intersection point of the normal direction and ground is the final aiming point. The intersection point coordinate under the condition of multiple radiation sources is showed in [5], which is: 


$$
x=\frac{\sum_{i=0}^{n} \sum_{k=0}^{n} E_{m i} E_{m k} \frac{\left(x_{k} z_{A}-x_{A} z_{k}\right)}{R_{k} \lambda_{k}} \cos \left(\phi_{i}-\phi_{k}\right)}{\sum_{i=0}^{n} \sum_{k=0}^{n} E_{m i} E_{m k} \frac{\left(z_{A}-z_{k}\right)}{R_{k} \lambda_{k}} \cos \left(\phi_{i}-\phi_{k}\right)}
$$

(1)

$$
y=\frac{\sum_{i=0}^{n} \sum_{k=0}^{n} E_{m i} E_{m k} \frac{\left(y_{k} z_{A}-y_{A} z_{k}\right)}{R_{k} \lambda_{k}} \cos \left(\varphi_{i}-\varphi_{k}\right)}{\sum_{i=0}^{n} \sum_{k=0}^{n} E_{m i} E_{m k} \frac{\left(z_{A}-z_{k}\right)}{R_{k} \lambda_{k}} \cos \left(\varphi_{i}-\varphi_{k}\right)}
$$

(2)

In the type: Emi and Emk are the electrical field peak of the electromagnetic waves radiated by $\mathrm{i}$-th and $\mathrm{k}$-th radiation source and $\lambda_{k}$ is the wavelength of electromagnetic wave radiated by $\mathrm{k}$-th radiation source. There is $\mathrm{i}=0,1,2, \ldots \mathrm{n}$, $\mathrm{k}=0,1,2, \ldots \mathrm{n}$, while $\mathrm{n}$ is the number of decoys. And there is:

$$
\varphi_{i}=\omega_{i} t-\frac{2 \pi R_{i}}{\lambda_{i}}+\varphi_{0 i}
$$

\section{(3)}

In the type: $\omega_{i}$ stands for angular frequency of i-th radiation source and $\omega_{0 i}$ stands for the initial phase. Rk stands for the distance from k-th radiation source to ARM. $\left(x_{A}, y_{A}, z_{A}\right)$ is the space coordinate of ARM.

But the main lobe of fire-control radar is too thin to be captured, so ARM locates the fire-control radar by its side lobe, usually the first side lobe. Then, the electrical field peak of radar's microwave at the seeker should be $\mathrm{p} 0 * \mathrm{Em} 0$, while p0 is the ratio of first side lobe and main lobe. To cover the whole air space, decoy adopts the omnidirectional antenna. The ratio of side lobe and main lobe of omnidirectional antenna is $\mathrm{pi}=1, \mathrm{i}=1,2, \ldots, \mathrm{n}$. Take the ratio into Eq.1 and Eq.2:

$$
x=\frac{\sum_{i=0}^{n} \sum_{k=0}^{n} p_{k} E_{m i} E_{m k} \frac{\left(x_{k} z_{A}-x_{A} z_{k}\right)}{R_{k} \lambda_{k}} \cos \left(\phi_{i}-\phi_{k}\right)}{\sum_{i=0}^{n} \sum_{k=0}^{n} p_{k} E_{m i} E_{m k} \frac{\left(z_{A}-z_{k}\right)}{R_{k} \lambda_{k}} \cos \left(\phi_{i}-\phi_{k}\right)}
$$

(4)

$$
y=\frac{\sum_{i=0}^{n} \sum_{k=0}^{n} p_{k} E_{m i} E_{m k} \frac{\left(y_{k} z_{A}-y_{A} z_{k}\right)}{R_{k} \lambda_{k}} \cos \left(\varphi_{i}-\varphi_{k}\right)}{\sum_{i=0}^{n} \sum_{k=0}^{n} p_{k} E_{m i} E_{m k} \frac{\left(z_{A}-z_{k}\right)}{R_{k} \lambda_{k}} \cos \left(\varphi_{i}-\varphi_{k}\right)}
$$

To accomplish the power combining effectively, the frequency of decoy and radar must always be the same, which means $\lambda_{i}=\lambda_{k}$. Some radar is equipped 
with warning system, which can detective ARM in real time. By controlling the radiate time of decoy signal, the phase of decoy signal and radar signal can be the same at ARM and the decoying effect can be promoted, as in [6] and [7].

Then, there is $\varphi_{i}=\varphi_{k}$. For ARM is locating fire-control radar through its side lobe, the possibility can be decreased only if the main lobe of decoy is near to the side lobe of radar to hide the later one. Decoy power is set as 1.3 times of radar's average side lobe in [8], which is very closely to radar's first side lobe. There is $p_{i} E_{m i}=p_{k} E_{m k}$, and the Equation can be simplified as:

$$
x=\frac{\sum_{i=0}^{n} \sum_{k=0}^{n} \frac{x_{k}}{R_{k}}}{\sum_{i=0}^{n} \sum_{k=0}^{n} \frac{1}{R_{k}}}
$$

(6)

$$
y=\frac{\sum_{i=0}^{n} \sum_{k=0}^{n} \frac{y_{k}}{R_{k}}}{\sum_{i=0}^{n} \sum_{k=0}^{n} \frac{1}{R_{k}}}
$$

As ARM is approaching radiation sources and arrives at the critical resolution point, some single radiation source is distinguished. Then ARM chooses the single radiation source or the power center of the rest radiation sources randomly to trace until end.

\section{Three-Source Anomalistic Layout Simulation}

Avoiding the research style of fixing a certain layout, a new decoy layout model covering all layouts is designed in this paper.

Simulation Interval. Three-source decoy system is adopted in simulation. Set the distance between the two decoys fixed and make the center of their linked line as the origin to build spatial rectangular coordinate system. The two decoys are set on $\mathrm{x}$-axis and the coordinate of radar changes by a certain order. While radar changes its coordinate, the shape of decoy system changes too. Every shape is simulated for 1000 times. The distance between the two decoys is set $300 \mathrm{~m}$, then their coordinate are $(-150,0,0)$ and $(150,0,0)$. Limit the $\mathrm{x}$ interval and $\mathrm{y}$ interval at $[-400,400]$. For the symmetry of coordinate system, space on first quadrant is only simulated.

Missile Parameter. Harm missile is chosen as the decoying target and its

Performance parameter is as follows: critical resolution angle $\Delta \theta_{R}=10^{\circ}$, angle measuring error is $1^{\circ} \sim 3^{\circ}$, maximum lateral overload coefficient $n=10$, and the maximum flying speed is $3 \mathrm{Ma}(1020 \mathrm{~m} / \mathrm{s})$, as in [9] and [10]. The original position in coordinate system changes from $5 \mathrm{~km}$ to $10 \mathrm{~km}$ randomly and always 
pointing to the power center of the three sources. For the top air attack style is adopt in modern ARM, the pitch angle should be set more than $45^{\circ}$ in initialization.

Decoying Success Condition. When ARM arrives at the ground and explored, it will cause a sector killing surface in front of it. The anterior killing distance is about $30 \mathrm{~m}$, while the rear killing distance is more than $10 \mathrm{~m}$. Being different from active decoy, radar also need effective working range about $20 \mathrm{~m}$ to receive signal. So, the effective save distance should be $60 \mathrm{~m}$. To ensure system safety and normal operation, the decoying success condition in simulation is that the distance between falling point of ARM and any radiation source is more than $80 \mathrm{~m}$.

The simulation process is shown in Fig. 2.

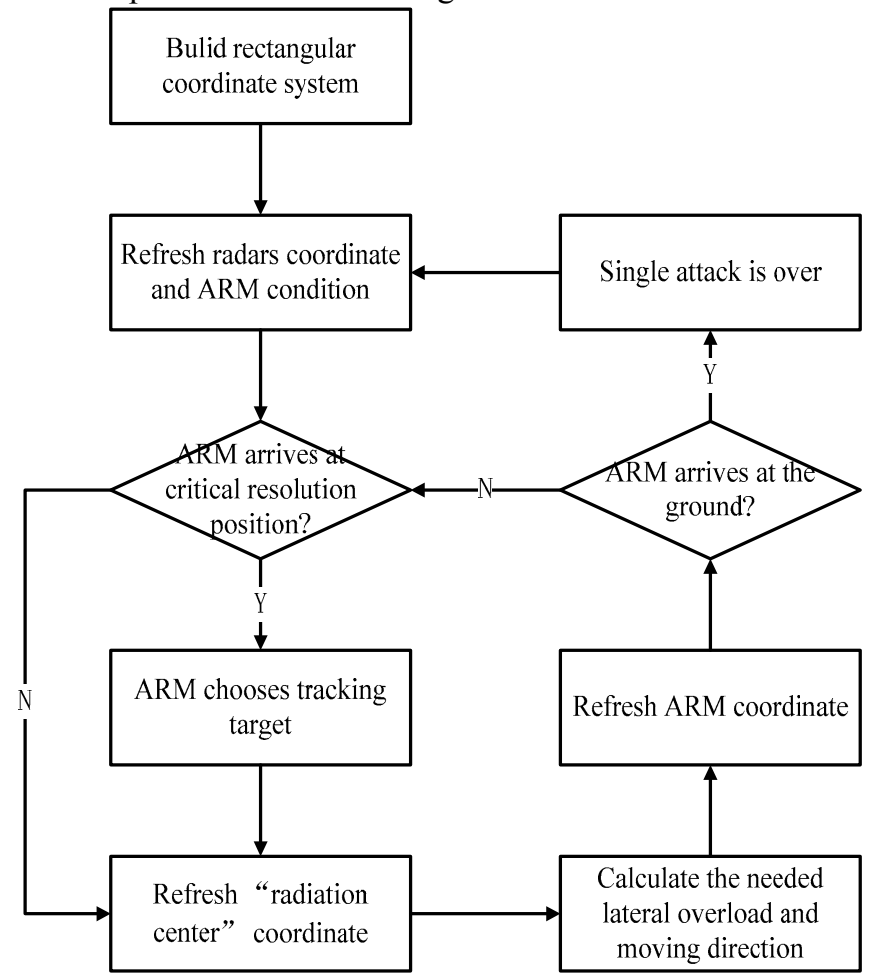

Figure 2. Process of decoying ARM.

\section{Simulation Result Analysis}

By modeling and simulation, the relationship between radar's position and decoying success rate is obtained and shown with 0.1 interval contours in Fig. 3. 


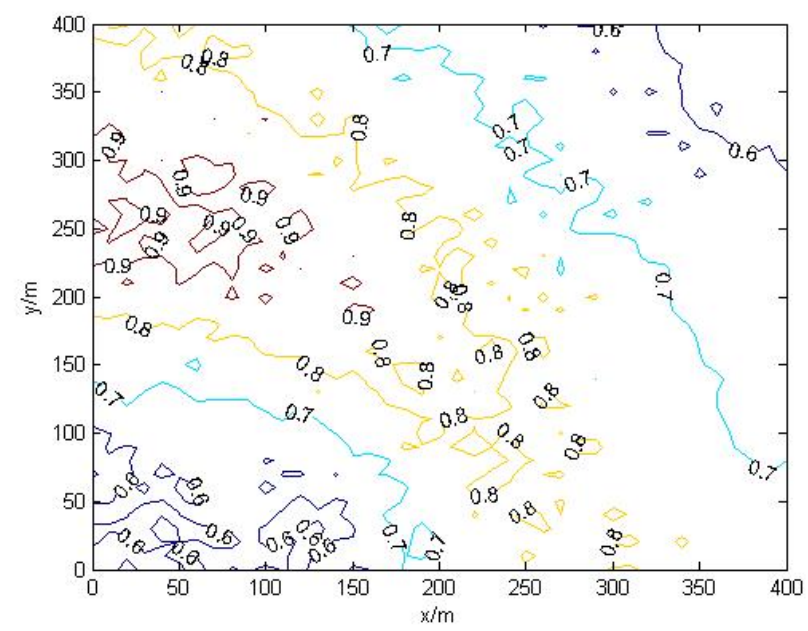

Figure 3. Relationship between success rate and radar's position.

Some conclusions can be obtained from Fig.3.

Anomalistic Annular Distribution. When radar's position changes at the set reign, the decoying success rate changes from 0.6 to 0.9 . With $\mathrm{x}$ coordinate and $\mathrm{y}$ coordinate increase by any linear relationship, the decoying success rate always increase then decrease. Then a conclusion can be obtained that the decoying success rate is related to the distance between radar to the center of two decoys linked line. In general, decoying success rate shows an anomalistic annular distribution making origin the center. With radar's coordinate is coming close to $\mathrm{x}$-axis, the band of the annulus becomes lager. And the condition of $\mathrm{y}$-axis is just on the opposite.

Changing Trend. With radar's position is moving away from the origin in different ways, the changing conditions of decoying success rate are relevant different.

When radar's position is moving away from the origin by $\mathrm{x}$-axis, the decoying success rates are low in general. Except very few positions can reach 0.8 , the decoying success rates are mostly from 0.6 to 0.8 and changes slowly. Especially when radar's position moves between the two decoys, radar is damaged badly. Because all radiation sources are decoying ARM to attack the region near the origin and the distance between radiation sources is not large enough.

When radar's position is moving away from the origin by y-axis, the decoying success rates are mostly from 0.6 to 0.9 and many of them are over 0.9 . The decoying success rate changes fast. The three sources present an isosceles triangle. When radar's position is between $230 \mathrm{~m}$ and $320 \mathrm{~m}$, the three sources approximately present an equilateral triangle and the decoying success reaches to the top. The high efficiency of equilateral triangle layout is confirmed.

The above analysis is the two extremely conditions. When radar's position is not on $\mathrm{x}$-axis or $\mathrm{y}$-axis, the decoying success rate changes between the two 
extremely conditions. And with the radar moving away from the origin, the influence of radar's orientation is decreasing.

Mathematic Model. The simulation result is obtained under the limited condition that the distance between the two decoys is $300 \mathrm{~m}$. To eliminate the influence of the limited condition, models under different distance setting should be simulated. But the distance cannot be too small. Otherwise, radiation sources are still in ARM's killing range, in spite of decoying well. The distance cannot be too large too, because ARM will distinguish the position of single radiation source. So, the distance is set from $200 \mathrm{~m}$ to $400 \mathrm{~m}$. Models are simulated and analyzed and the decoying success rate changing trend is always the same. The mathematic model of decoying success rate is as follows:

$$
\frac{x^{2}}{[(2 s-0.7) \cdot l]^{2}}+\frac{(2 y)^{2}}{[(2.7 s-0.9) \cdot l]^{2}}=1
$$

$$
\frac{(2 x)^{2}}{[(7.9-6 s) \cdot l]^{2}}+\frac{y^{2}}{[(4-3.3 s) \cdot l]^{2}}=1
$$

In the type: $\mathrm{x}$ and $\mathrm{y}$ are radar's coordinate, and 1 is the distance between the two decoys, ranged from $200 \mathrm{~m}$ to $400 \mathrm{~m}$. The s stands for decoying success rate, ranged from 0.6 to 0.9 . The annulus of decoying success rate is composed of inner boundary and outer boundary. Eq. 8 and Eq.9 are the mathematic model of the two boundarys. When inner boundary and outer boundary intersects, the annulus can't be formed. Such as when $s=0.9$, the region is only distributed near y-axis.

Recommended Configuration. The recommended configuration of fire-control radar three-source active decoy layout is put forward. Locate fire-control radar near the perpendicular bisector of the two decoys and set the distance at 0.771 to 1.071, while 1 is the distance between the two decoys. Meanwhile, the angle between the perpendicular bisector and the linked line form radar to the center of the two decoys should be set less than $20^{\circ}$. Then the decoying success rate is about 0.9 .

\section{Conclusion}

Based on the active decoy principle and making influential factors, like fire-control radar antenna' characteristic, signal Synchronization and power combination into consideration, three-source active decoy layout is studied and the coordinate formula of ARM tracing point is transformed and simplified. And a new layout model covering the anomalistic layout is designed. By massive simulations, the relationship between decoying success rate and the radar's position in decoy system is obtained. The recommended configuration of three-source decoy system is also put forward, and it will support the battle application of fire-control radar active decoy. 


\section{References}

[1] Wang Zhanfeng, Lv Xuliang, Wu Xiaolian: Journal of PLA University of Science and Technology. Vol. 8(2007), p. 270-273.

[2] Ai Mingshun, Ma Hongguang: Modern Radar. Vol 32(2010), p. 13-16.

[3] Li Liujing, Ma Yanheng, Liu jing: Computer Measurement \& Control. Vol 19(2011), p. 2187-2196.

[4] Min Tao, Jiang Wenli, Zhou Yi-yu: Aerospace Electronic Warfare. Vol $3,(2000)$.

[5] Hu Fang, Liu Yuchi, Tuo Xinyu: Computer Emulation. Vol 27(2010), p. 31-35.

[6] Xiao Tao, Tang Ziyue, Ji Jingxiang: Modern Radar. Vol 29(2007), p. 9-11.

[7] Zhou Weiguang, Luo Jirun: Journal of Electronics \& Information Technology. Vol 32(2010), p.1370-1376.

[8] Miao Xiumei, Wang Juan, Xu Hailong: Radar Science and Technology. Vol 35(2012), p. 59-65.

[9] Ren Gangqiang: Northwestern Polytechnical University, 2005.

[10] LINENG: IEEE Transactions on Aerospace and Electronic Systems. Vol 31(1995), p. 1120-1127. 